\title{
Les coopératives des vendeuses de marché: Accorder crédit aux
} femmes

Judith Bruce

Population Council

Follow this and additional works at: https://knowledgecommons.popcouncil.org/departments_sbsr-pgy

Part of the Family, Life Course, and Society Commons, Gender and Sexuality Commons, International Public Health Commons, and the Regional Economics Commons How does access to this work benefit you? Let us know!

\section{Recommended Citation}

Bruce, Judith. 1984. "Les coopératives des vendeuses de marché: Accorder crédit aux femmes," SEEDS no. 3. New York: Population Council. 


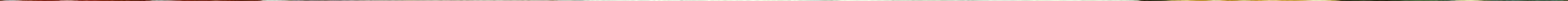


SEEDS est une série de brochures publiée en réponse aux demandes d'information venant du monde entier sur des idées de programmes innovatrices et pratiques, réalisées par et pour des femmes aux revenus faibles. Le but de ces brochures est de diffuser l'information et de stimuler la création de nouveaux projets, fondés sur les expériences positives de femmes qui travaillent pour améliorer leur propre statut économique et celui des autres. Les projets décrits dans ce numéro comme dans le reste de la série ont été choisis du fait qu'ils procurent aux femmes un revenu en espèce et les font participer activement aux prises de décisions comme aux profits. Ces projets sont bâtis suivant des critères économiques solides et permettent de surmonter avec succès des obstacles courants. Ces rapports n'ont cependant pas été conçus pour être suivis à la lettre car chaque effort de développement doit faire face à des ressources et des problèmes quelque peu différents. En revanche, ils relatent l'histoire d'une idée et sa mise en application avec l'espoir que les leçons apprises se révèleront utiles dans des environnements variés. C'est également pour être portés à l'attention de ceux qui détiennent les pouvoirs de décision qu'ils ont été rédigés et leur montrer que des projets générateurs de revenus, pour et par les femmes, sont viables et jouent un rôle important dans le développement.

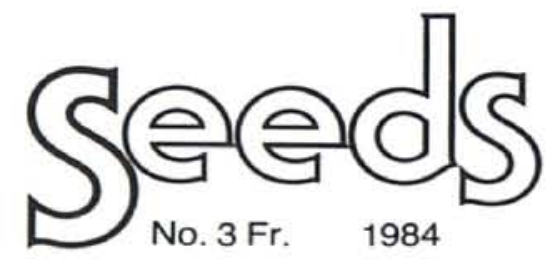

La publication de SEEDS est administree par The Population Council. Les criteres de publication sont détermines par le Comité de Rédaction de SEEDS: Kristin Anderson (Center for Public Advocacy Research), Judith Bruce (The Popula-

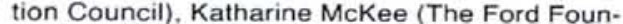
dation), Jill Sheffield (The Carnegie Corporation) et Ann Leonard (Editeur). 


\title{
Les Coopératives des Vendeuses de Marché: Accorder Crédit aux Femmes.
}

\author{
Par Judith Bruce
}

\section{Introduction}

Les femmes d'Amérique Centrale, comme celles de la plupart d'autres pays, sont économiquement actives, en particulier dans le domaine de l'agriculture de subsistance et le petit commerce. Cependant, et à l'image d'autres femmes ailleurs, leurs contributions à l'économie des foyers, des communautés et de la nation restent en général ignorées par les institutions politiques et financières. Au Nicaragua, les vendeuses de marché jouent un rôle important dans la vie d'une grande partie de la population du pays: Elles offrent des produits à bas prix, sur des lieux de vente pratiques et populaires, personnalisant et réaffermissant des liens établis par des rencontres quotidiennes avec leurs clientèles. Pareillement à n'importe quelle autre entreprise, il leur est souvent nécessaire de pouvoir disposer d'argent liquide pour poursuivre leurs activités. Toutefois, perçues en marge des principaux secteurs de l'activité économique nationale, obtenir des crédits leur est très difficile. En 1972, FUNDE, la Fondation Nicaraguayenne de Développement (Fundacion Nicaraguense de Desarrollo), I'un des deux programmes patronnés par l'Institut Nicaraguayen de Développement (INDE), prit connaissance du problème. Les pages qui suivent sont une description des efforts de FUNDE pour répondre à ce besoin par la création et la mise en place de coopératives d'épargne et de crédit. Ce rapport insiste autant sur les aspects humains que ceux financiers et techniques du projet. Le succès obtenu s'explique par la manière dont les coopératives se sont formées, respectant les systèmes de valeurs, coutumes et traditions des vendeuses et se servant des multiples relations interpersonnelles, subtiles et complexes, établies au cours des années. Essentiellement, ces coopératives ont permis aux femmes d'apprendre à se servir au maximum et à utiliser au mieux leurs propres ressources en leur procurant information, expérience et encouragement. 


\section{L'apprentissage}

Les vendeuses de marché ont besoin d'argent liquide pour de nombreuses raisuns: Acheter les produits et simples objets de fabrication qu'elles revendent, assurer leurs frais de transport, louer leurs emplacements, payer pour l'eau, l'électricité, les "cargadores" qui transportent leurs biens sur les lieux de vente, et l'utilisation des toilettes publiques. A Managua, la capitale, les frais d'exploitation pour un emplacement de taille moyenne s'élèvent approximativement de 100 à 1000 Cordobas par jour (10 à 100 Dollars US). Dans des villes plus petites, de plus modestes opérations reviennent sans doute moins cher mais cela ne diminue pas la nécessité de pouvoir accéder à des sources d'argent liquide. Dans la majorité des cas, les revenus sont minces et les femmes ne peuvent mettre de l'argent de côté que rarement. Elles gèrent leurs affaires le plus souvent au jour le jour. Tout cela revient à dire que, pour garder leurs commerces à flôt, elles doivent emprunter fréquemment. En outre, emprunter leur est impératif si elles veulent agrandir leurs affaires et augmenter leur volume pour faire face à la compétition des supermarchés et autres systèmes de marketing et de distribution.

En théorie, les vendeuses de marché bénéficient de trois possibilités de crédit: Les banques ou caisses de crédit, leurs familles et amis, et les prêteurs professionnels, les "prestamistas". En réalité, prêts et crédits bancaires sont difficiles à obtenir car la plupart des banques locales refusent d'une part d'administrer la gestion des petites sommes demandées et, d'autre part, estiment que les entreprises de ces femmes n'offrent pas de garanties suffisantes ou acceptables. Au reste, les banques exigent un co-signataire et seul un homme, le mari de préférence, est présumé pouvoir se porter garant. Le système perpétue donc le mythe que les femmes restent dépendantes de leurs maris pour la satisfaction de leurs besoins financiers, même si elles représentent en fait le principal ou unique soutient économique du ménage. Les amis ou la famille, quant à eux et s'ils le peuvent, n'ont que peu d'argent à prêter. Restent les "prestamistas" qui, généralement, accordent des prêts à court-terme (à 30-60 jours), avec un taux d'intérêt mensuel de $10 \%$ et annuel de 120 a $240 \%$

FUNDE, connaissant le problème et sachant que les femmes avaient besoin d'emprunter à des taux plus raisonnables, pensa que la Fondation pouvait les aider. Elle ne parvint à une solution solide qu'après plusieurs tenta- tives dont la première fut un échec. FUNDE commenca par ouvrir une caisse de prêts sur le Marché Central pour permettre aux nombreuses commerçantes y travaillant d'y obtenir des prêts au taux abordable de $1 \%$ par mois ou $12 \%$ par an. En dépit de cela, les femmes, pour beaucoup, continuèrent leurs transactions habituelles avec les "prestamistas", à des taux dix fois supérieurs à ceux proposés par FUNDE. Pour déterminer les causes de cet échec, FUNDE engagea deux des "prestamistas". Ces derniers révélèrent plusieurs raisons pour lesquelles les vendeuses hésitaient à se tourner vers la Fondation pour emprunter: Les bureaux étaient trop formels, la manière d'opérer, étrange et impersonnelle et, pour citer l'un des prêteurs, "après une longue journée sur le marché, une femme pourrait facilement attraper froid içi", se référant à l'air climatisé des bureaux. En contraste, le prêteur parle librement, sans contrainte et de façon intime à sa cliente dont il connait bien la situation et la personne. Sa présence quotidienne sur le marché est un avantage précieux pour la femme, permettant à celle-ci de le rembourser un peu chaque jour. De plus, en cas de besoin urgent, elle peut généralement aller le trouver et obtenir de l'argent en dehors des heures régulières. Clairement, pour pouvoir concurrencer les "prestamistas" de façon efficace, FUNDE se devait de changer sa stratégie. Se

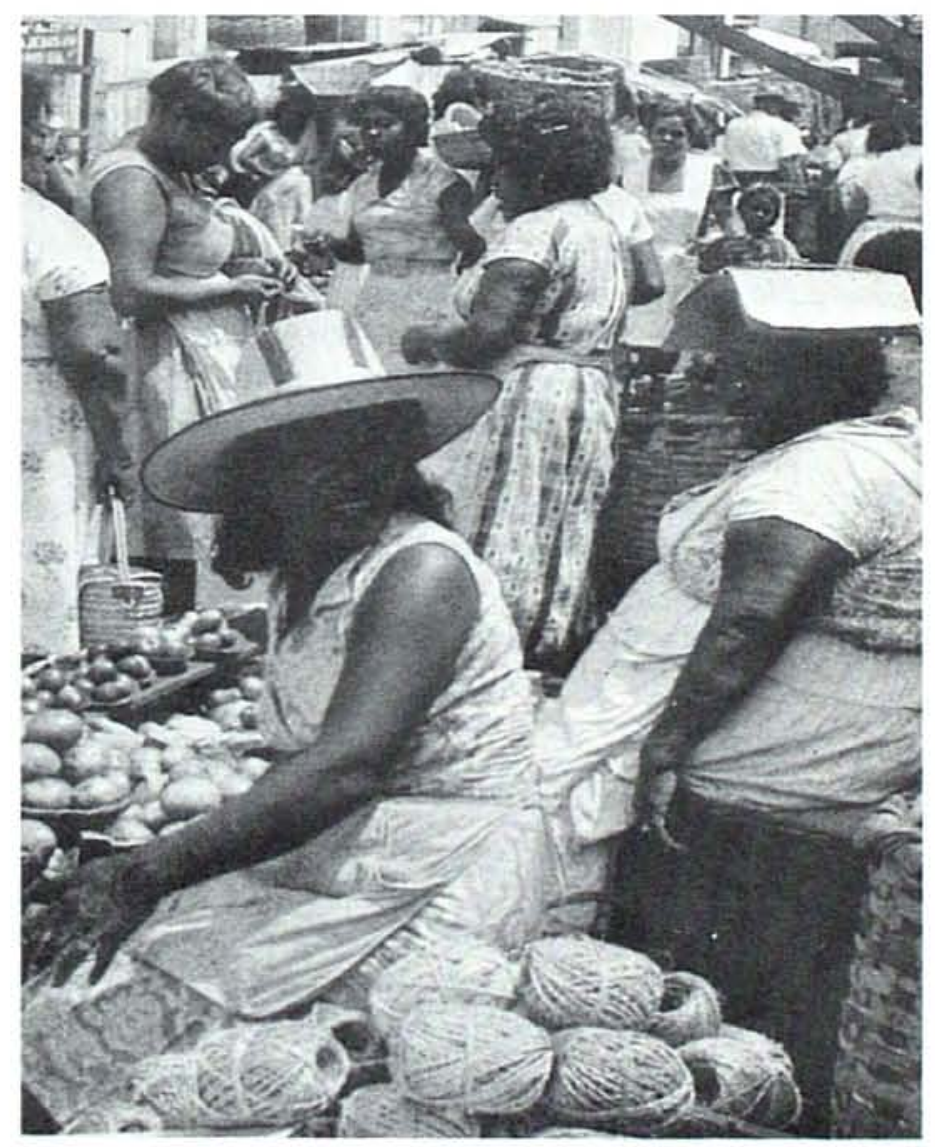


servant de la leçon apprise et de son expérience dans la formation de coopératives pour une population à faible revenu, FUNDE décida d'essayer un système où les femmes mettraient en place leurs propres organisations de crédits dans leurs communautés, opérations qu'elles finiraient par diriger elles-mêmes: Une coopérative d'épargne et de crédit pour les vendeuses de marché. De telles coopératives, pour des femmes qui, souvent, ne jouissent pas d'une image sociale élevée et n'appartiennent que rarement à des institutions reconnues, pourraient non seulement les faire participer à l'expansion et la diversification économique mais, de plus, leur permettraient d'effacer leurs dettes, encourageraient l'épargne et amélioreraient leur statut social.

\section{Créer Une Coopérative}

A long-terme, le but de FUNDE est d'offrir à toutes les commerçantes de marché du Nicaragua la possibilité de faire partie d'une coopérative locale d'épargne et de crédit. La Fondation décida pour débuter de choisir des villes de marché de plus de 2.500 habitants. Elle avait déjà entrepris des projets dans plusieurs villes de taille similaire et était relativement bien connue. Utilisant son expérience et progressant au coup par coup, FUNDE suivit les différentes étapes nécessaires à la formation de coopératives.

Les premières personnes rencontrées dans ces villes sont leurs responsables, notables et dirigeants. S'ils se montrent favorables à de futures réunions, un groupe de base, comptant de cinq à trente membres potentiels, est constitué, sous les conseils et recommendations des autorités locales et de l'équipe déléguée par FUNDE. Au cours de ces réunions, on explique et discute la signification, les structures, fonctions et avantages des coopératives. FUNDE souligne qu'une coopérative n'est pas seulement un moyen d'obtenir des crédits. Les adhérentes doivent avoir épargné avant de pouvoir emprunter (En règle générale, les prêts consentis n'excèdent pas plus de trois fois les sommes déposées et ne sont accordés que 45 jours après l'ouverture d'un compte). Un autre avantage présenté, bien que moins tangible, est le rôle d'une coopérative dans la résolution de problèmes économiques par une prise en charge et une action collective.

Les premières rencontres durent environ deux heures. Elles sont tenues aux alentours immédiats du marché pendant une dizaine de jours, après quoi peut-être 100 femmes sont venues écouter et discuter. Pour la plupart,

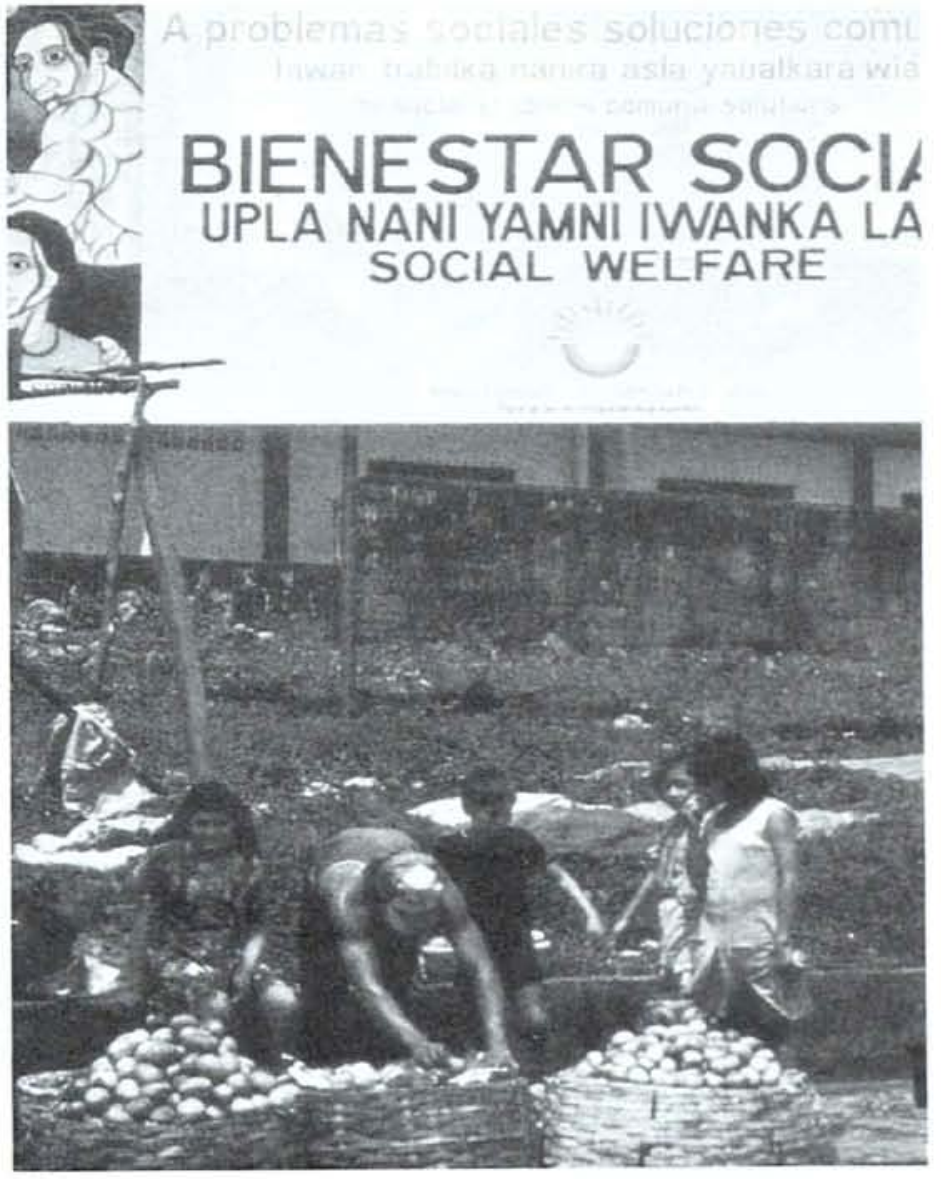

elles viennent d'abord par curiosité. Habituellement, les premières à se présenter sont relativement plus aisées que le reste, même si elles sont également fortement endettées auprès des "prestamistas". C'est le solide enthousiasme de ces membres potentiels qui réussit ensuite à convaincre les autres. De nombreuses réunions sont nécessaires pour attirer suffisemment de monde et établir une bonne crédibilité ainsi que pour assurer la pleine compréhension des informations communiquées. L'équipe de FUNDE et le groupe de base expliquent et réexpliquent mille et une fois comment une coopérative fonctionne. C'est une étape essentielle si l'on veut qu'une coopérative bénéficie de la confiance des habitants. L'acceptation du concept d'une coopérative est plus lente dans des communautés où de précédentes tentatives ont échoué et où les "prestamistas" font courir le bruit que la coopérative volera l'argent de ses membres.

Quand une trentaine de femmes ou plus ont décidé de s'associer en coopérative, FUNDE les aide à s'occuper des déclarations et formalités légales. II est primordiale pour une coopérative de posséder une existence légale si elle veut pouvoir indépendemment recevoir et distribuer des fonds, établir des relations avec d'autres institutions (telles les 


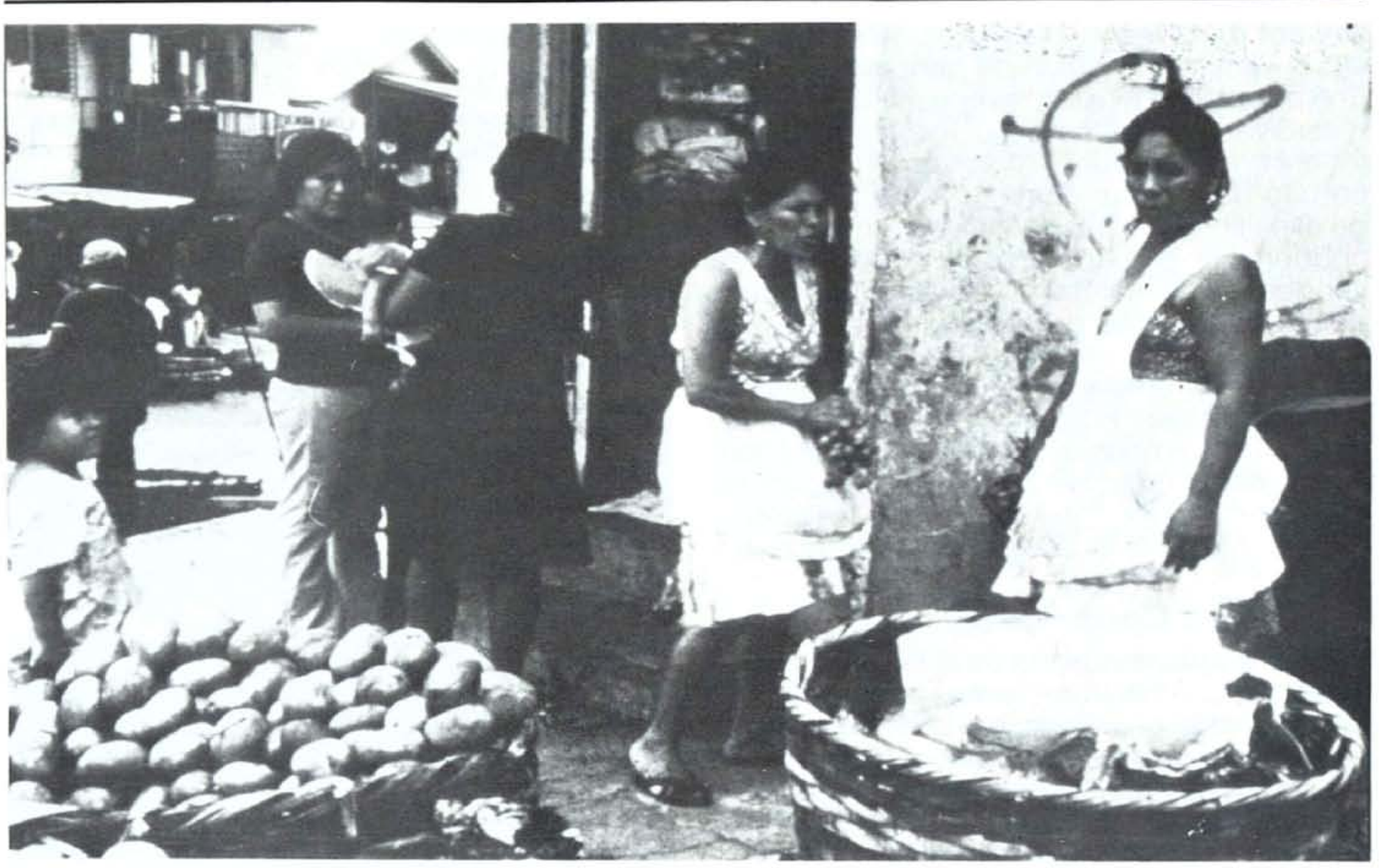

banques), jouer un role de pression si nécessaire et enfin, se détacher entièrement de FUNDE et devenir autonome. Chaque étape de ces formalités est expliquée et commentée en détail car beaucoup de ces femmes sont illettrées et peu d'entre elles sont familières avec les procédures gouvernementales et le système législatif. Une coopérative existe officiellement lorsque tous les documents légaux requis ont été signés, onze directrices ont été élues par les participantes et quand chaque adhérente a acheté une part de la coopérative pour 10 Cordobas et payé 10 autres Cordobas pour les frais d'administration et 2 Cordobas pour un livret de compte. II faut compter de deux à trois mois pour compléter toutes les procédures légales indispensables.

Pendant ce temps, on loue un bureau dans un endroit pratique, en général près du marché. II ressemble à n'importe quel autre bureau de la ville, meublé simplement et disposant souvent de toilettes à l'extérieur. FUNDE fait don des meubles et de l'équipement: Une armoire de classement, un fichier, une machine à écrire, un bureau, une chaise et une calculatrice. Tout cela prend une valeur symbolique importante pour la nouvelle coopérative. Puis FUNDE accorde un prêt global de 10.000 Dollars US à $10 \%$ d'intérêt annuel pour permettre à la coopérative de démarrer le procédé d'emprunt. Un autre prêt important est établi pour donner les moyens à la direction et à l'organisation d'assurer 18 mois de loyer et le salaire d'une directrice générale (22.248 Cordobas, soit 3.000 Dollars US). Ce prêt est gratuit. En analysant les différentes causes d'échec des coopératives, échec financier ou échec dans l'enrôlement et la rétention de nouveaux membres, FUNDE s'aperçut que la plus grosse erreur commise était souvent le manque de direction qualifiée. En conséquence, dès qu'une directrice générale est engagée, elle suit immédiatement un programme de formation spécialisée. Cette personne est sélectionnée en commun par les directrices élues et par FUNDE, leur choix se basant sur des rencontres en tête à tête et un test d'aptitudes professionnelles générales. Fréquemment les candidates sont de jeunes femmes possédant un diplôme d'études secondaires. Leur salaire est égal ou supérieur à celui versé pour un poste de supervision en usine ou dans des bureaux. Un aspect unique du rôle de la directrice générale est qu'elle répond de son travail directement aux membres de la coopérative et non à FUNDE.

Un second facteur expliquant d'autres échecs précédents était le manque de programme de formation adéquat et soutenu. La contribution essentielle de FUNDE au succès d'une nouvelle coopérative n'est pas l'argent, puisqu'il est éventuellement remboursé, mais 
son assistance technique dans le développement des qualités nécessaires à une coopérative pour devenir autonome. Deux types de stage de formation sont utilisés, l'un pour les directrices et directrices générales, l'autre, pour le reste des adhérentes. Les premières sont habituellement envoyées à Managua pour donner au programme plus de prestige et réduire au minimum les distractions. Les autres suivent leur stage sur place, dans les bureaux de la coopérative ou à proximité. On se sert de deux sortes de méthode d'enseignement: L'étude de cas et l'analyse de documents. Pour la première, on présente à de petits groupes plusieurs exemples de situations qui pourraient vraisemblablemnt se produire dans la vie d'une coopérative. Chaque groupe détermine le problème et ses causes et décide ensuite de la façon dont il devrait être résolu. Puis, chaque groupe présente ses recherches à l'ensemble de la classe qui commente, critique discute et, finalement, I'on tire les conclusions. Dans la deuxième méthode, on distribue des documents décrivant différentes coopératives. Les élèves prennent connaissance des faits en étudiant individuellement ou en discutant en groupe. On utilise aussi quelquefois une troisième méthode, maintenant que les coopératives deviennent plus courantes: l'étude d'une "coopérative modèle". Pour apprendre et assimiler le fonctionnement de leurs opérations, directrices et membres vont visiter et observer des coopératives bien dirigées, efficaces et productives.

A l'heure actuelle, directrices et membres du comité de direction doivent savoir lire et écrire. Mais du fait que beaucoup d'adhérentes sont illettrées, le matériel employé lors des stages de formation comprend de nombreuses illustrations qui représentent les concepts de base et provoquent la discussion. On se sert d'images et de tableaux pour aider les participantes à repondre à des questions telles que: "Quel sera le but de la coopérative cette année?". Si le but recherché est de pouvoir compter 300 nouveaux membres, on produit un dessin éloquent des 300 nouvelles associées. De même, une illustration représentant les marchés tels qu'ils étaient auparavant et ce qu'ils peuvent devenir grâce à l'aide d'une coopérative bien organisée, aide les participantes à apprendre les avantages que I'on peut tirer du développement d'une coopérative. Les méthodes de formation mentionnées s'appuient sur des idées et des concepts familiers. Par exemple, le concept de la Sainte Trinité, dans la religion Catholique Romaine, (trois dans un) est utilisé pour démontrer qu'une coopérative est un tout, formé de trois groupes différents: Propriétaires, travailleurs et associés et chaque membre fait partie de ces trois groupes en même temps.

Les sessions de formation durent de trois à six jours. Cette période est suffisemment courte pour permettre aux femmes qui ont une famille de participer. Toutefois, on ne peut prétendre achever la formation complète des membres d'une coopérative en une seule série de sessions, et directrices et adhérentes suivent fréquemment des classes supplémentaires qui les préparent petit à petit à devenir autonomes. Apprendre un nouveau rôle et as-

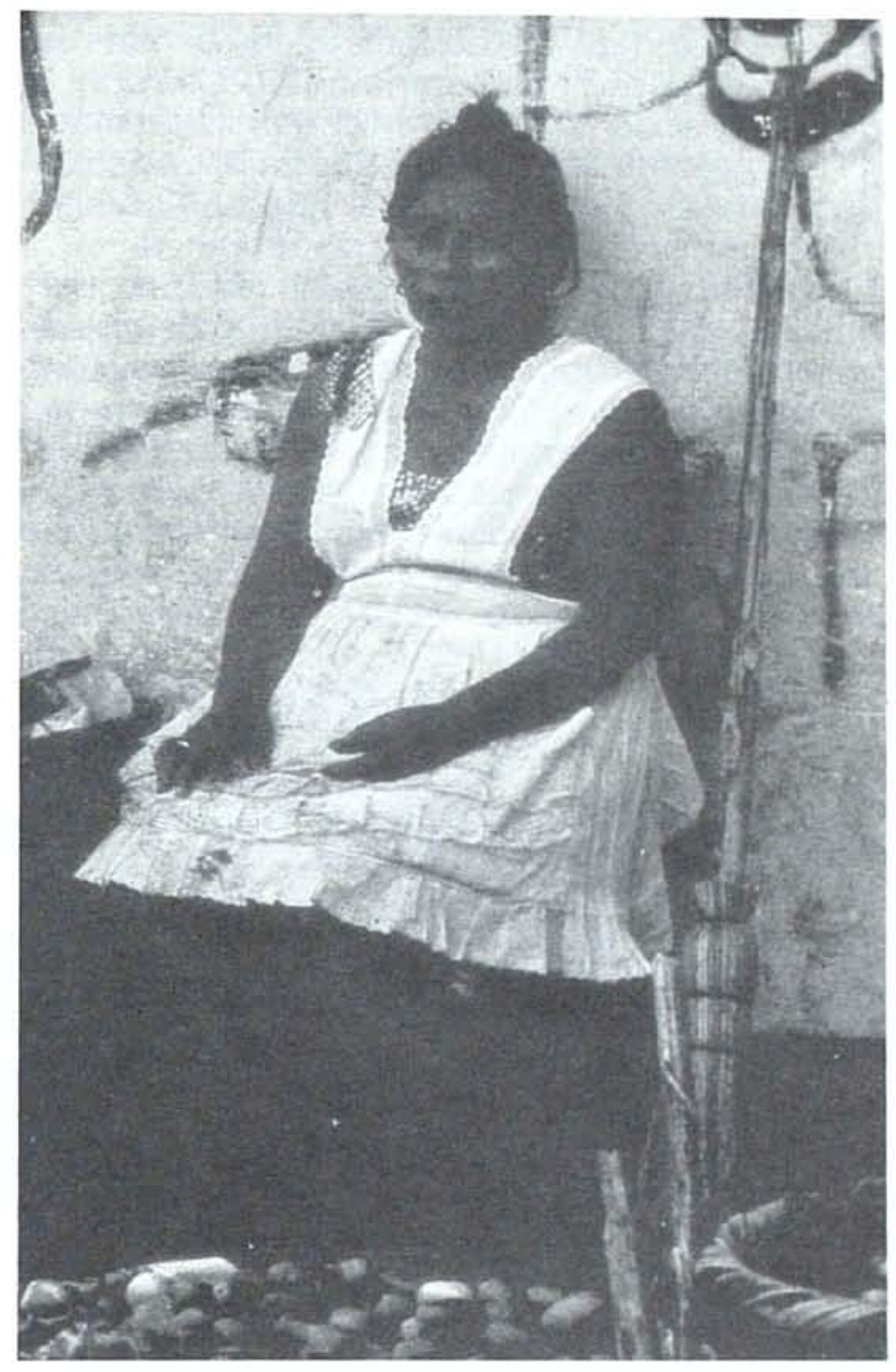

similer de nouveaux concepts sur soi-même prend du temps. Dès que la coopérative est implantée légalement, le travail commence. Au début, le nombre des inscriptions tourne autour de 30 à 50 personnes et n'atteint la marque des 200 ou 300 qu'après plusieurs années. La majorité des coopératives accueillent quiconque souhaite $y$ adhérer mais ce sont les membres qui, en persuadant d'autres femmes de joindre leur équipe, font augmenter 
la participation. Cela semble être un phénomène de groupe: Un nouveau groupe de femmes s'inscrira après que l'une d'entre elles, déjà membre de la coopérative, se soit déclarée satisfaite de son expérience.

Pour être considérée comme membre actif, une adhérente n'a pas nécessairement à participer aux assemblées générales annuelles ou à d'autre réunions mais elle doit avoir commencé à épargner ou contracté un prêt. L'âge varie de 18 à 80 ans, les directrices se rangeant habituellement dans la catégorie des 30-50 ans.

Les onze directrices, sélectionées par les membres, s'organisent en trois comités: 5 dans un Comité d'Administration, 3 dans un Comité de Crédit et 3 dans un comité dont la fonction est de contrôler et vérifier l'exactitude et l'honnêteté des deux précédents. Les structures d'une coopérative et le rôle des comités sont décrits en détail dans l'appendice I.

Chaque semaine, durant les premiers mois d'opération, on tient des assembleés générales auxquelles une représentative de FUNDE participe. Ces réunions sont ouvertes à tout le monde pour souligner la nature démocratique d'une coopérative et permettre aux informations de circuler dans la communauté. On distribue à toutes des brochures imagées illustrant les fonctions et les structures de la coopérative ainsi que les démarches à suivre pour obtenir un prêt (voir appendice II). Les brochures doivent être conservées et utilisées comme références. On les distribue également aux autres habitantes de la communauté qui ne sont pas encore membre de la coopérative, comme un moyen de les motiver à s'inscrire. De plus, on utilise de nombreux supports visuels pour la marche journalière de la coopérative, notamment de larges posters muraux indiquant le nombre des adhérentes ou le total des sommes déposées ou empruntées. Ces tableaux sont mis à jour chaque semaine et représentent une mesure tangible des progrès de la coopérative. Pouvant être comprises et assimilées facilement aussi bien par des illettrées que par d'autres, ces illustrations aident à combler le fossé entre les deux groupes. C'est un point crucial pour I'homogénéité et la cohésion de la coopérative.

En règle générale, une coopérative démarre lentement. La période d'organisation dure de un à trois mois. La première demande de prêt est soumise entre trois et six mois. Puis, quand le système et la façon dont il fonctionne ont été démontrés et sont largement assimilés, le nombre des demandes de prêt augmente de manière substantielle. Vient ensuite une période de stabilisation où la courbe des demandes varie de façon prévisible. Typique-

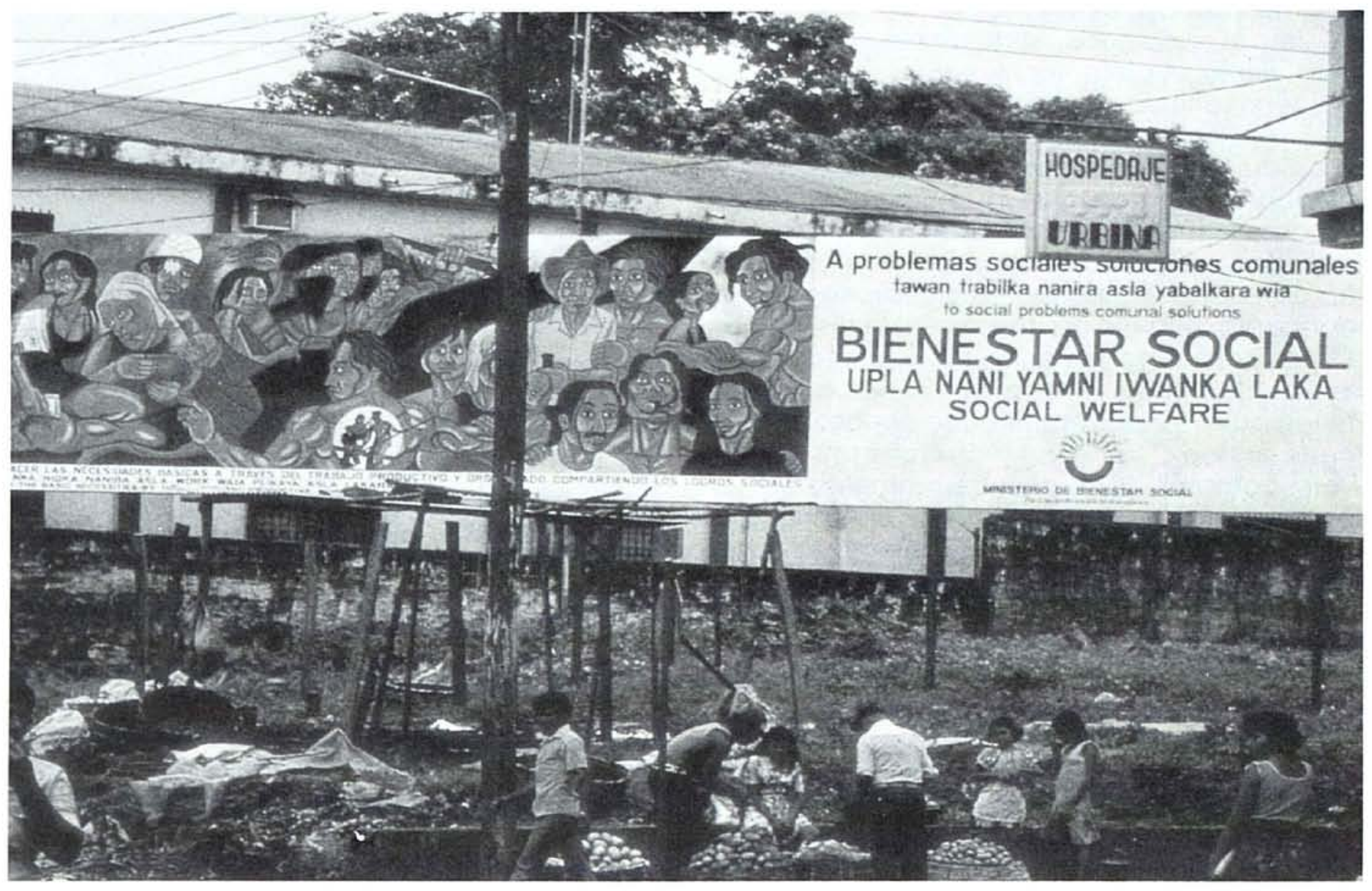


ment, une période d'épargne est suivie par une période d'emprunt.

\section{Obtenir Un Prêt}

Quand une adhérente souhaite obtenir un prêt, elle s'adresse à la directrice générale qui remplit une demande indiquant la somme désirée, le but, l'étalage des paiements proposé ainsi que le montant des épargnes réalisées par la candidate à ce jour. Puis, le dossier est étudié par le Comité de Crédit qui se réunit au minimum une fois par semaine ou quelquefois, chaque jour. Le Comité entreprend une analyse détaillée des "risques" représentés par la candidate, se basant sur leurs connaissances de sa personnalité, le genre de travail qu'elle effectue, la façon dont elle le conduit et le rythme auquel elle peut produire ses revenus. Le Comité est supposé formé son jugement en s'appuyant sur ses propres perceptions et non celles de la directrice générale ou d'autres personnes. Une bonne analyse étudie chaque dossier attentivement, repousse la décision finale en cas de controverse, considère un prêt comme un droit et non un privilège et n'accorde pas de crédits au délà des moyens de la bénéficiaire.

Le Comité de Crédit joue un rôle important, tout spécialement lors des premiers mois d'existence de la coopérative. Ses membres alternent périodiquement pour éviter le favoritisme mais il compte toujours trois personnes. Appartenant le plus souvent à la même communauté que les candidates pour un prêt, les membres du Comité occupent une position où il leur est facile de savoir qui représente un bon ou un mauvais risque. De plus, cela peut aider à exercer un peu de pression pour être sûr qu'un emprunt sera remboursé. Les conditions formelles pour obtenir un prêt sont les suivantes:

1. Les candidates doivent avoir épargné pendant au moins six semaines avant de pouvoir soumettre leur première demande.

2. Tour prêt antérieur doit avoir été remboursé intégralement avant de pouvoir effectuer une nouvelle demande.

3. Les candidates ne peuvent obtenir un prêt supérieur à trois fois le montant de leurs épargnes.

4. Les prêts dont le montant dépasse 3000 Cordobas doivent être remboursés en un an. Les prêts de moins de 3000 Cordobas doivent etre remboursés en huit mois (il y a des exceptions).

5. Aucun membre ne peut emprunter plus de 8000 Cordobas.

6. Le taux d'intérêt est de $1 \%$.

7. Le co-signataire du prêt doit être un autre membre de la coopérative ou une personne possédant les ressources financières nécessaires à couvrir le montant du prêt.

8. Aucun membre n'est autorisé à se porter garant de plus de deux prêts simultanément.

9. Si un membre désire se retirer de la coopérative, un préavis de 30 jours est requis de facon à ce que toutes les transactions en cours (remboursements, etc.) puissent être completées et les sommes en dépôt, remboursées. Si une participante ne peut, malgré plusieurs requêtes, honorer ses paiements, elle est invitée à se retirer de la coopérative.

Environ 3\% des prêts ne sont jamais remboursés. 10 à $15 \%$ des plans de paiements doivent être réajustés. Les versements prévus tiennent compte des besoins individuels. Là où des différences dans les plans de remboursement peuvent parfois créer une certaine tension entre les participantes, il est cependant important pour les coopératives de rester flexibles. Ces remboursements doivent être échelonnés en fonction de ce que les femmes peuvent raisonnablement payer. Les "prestamistas" recoivent des paiements quotidiens. Les banques, d'un autre côté, estiment que le temps et les papiers requis pour ces transactions journalières sont trop accaparants. Les coopératives, elles, acceptent cette façon de paiement car beaucoup de leurs membres ne tiennent pas à garder trop d'argent avec elles de peur de se voir forcer par leurs familles à le dépenser. Au cours d'une journée moyenne, les bureaux de la coopérative reçoivent de cinq à trente visites par des membres qui viennent toucher leurs prêts, verser leurs paiements ou déposer de l'argent sur leurs livrets. Les heures d'ouverture sont de 9 heures du matin jusqu'à la fermeture du marché vers 4 ou 5 heures de l'après-midi. Tout le monde connait l'adresse de la directrice générale et peut ainsi aller la trouver en cas d'urgence. Entre deux visites, cette dernière s'occupe des comptes et autres tâches administratives dont elle a la charge.

Dès que la coopérative est bien lancée, ses trois comités (Crédit, Administration, Controle) se réunissent au moins une fois par semaine. L'ensemble des membres se réunit une fois par mois, à l'invitation de la directrice 


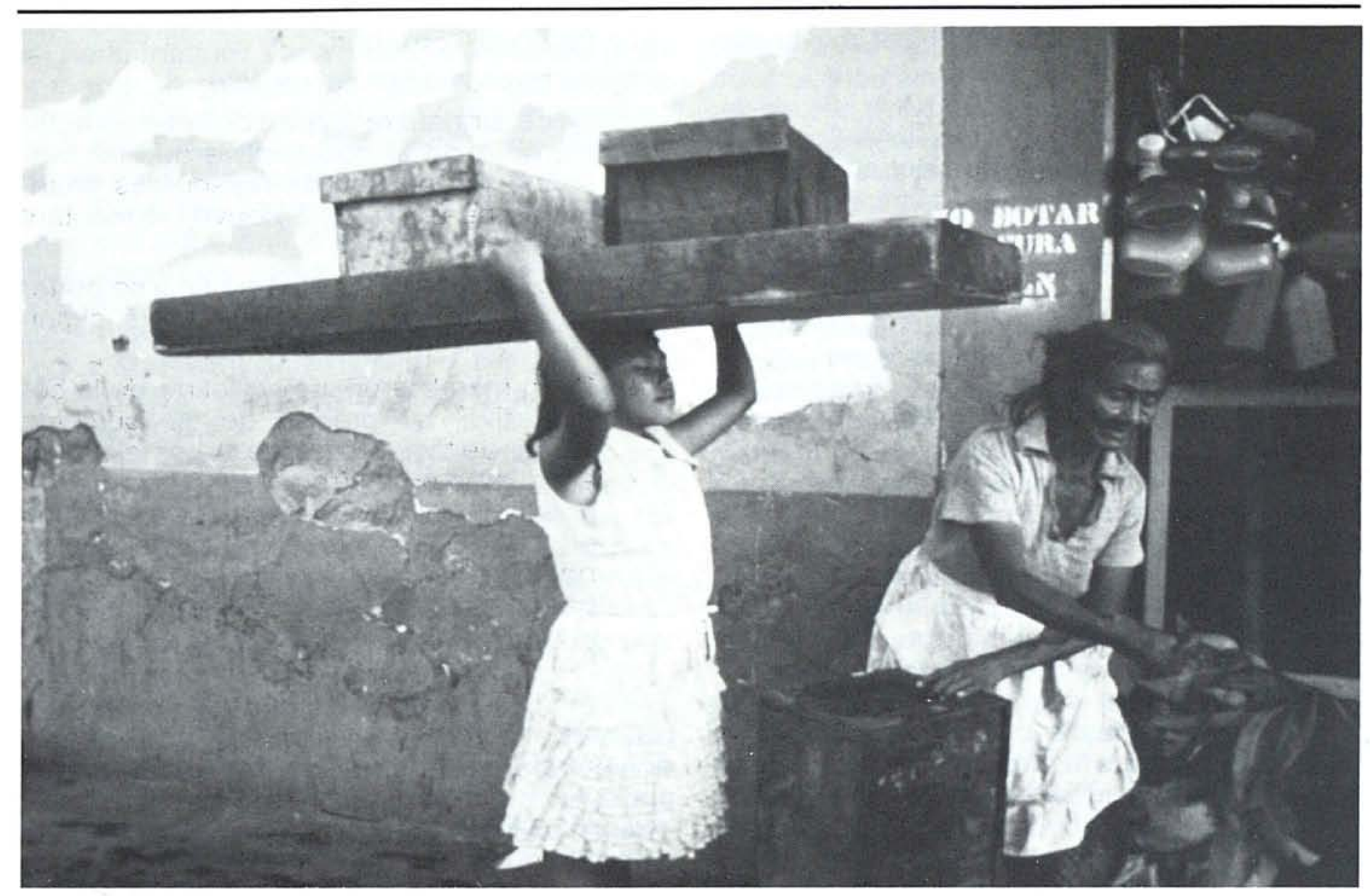

générale. On tient également une assemblée générale annuelle où la situation financière de la coopérative est analysée et des rapports écrits sont préparés par chaque comité. Ces rapports sont reproduits et distribués aux participantes. Ils représentent un important moyen de communication car ils offrent non seulement des informations précises et spécifiques mais, de plus, resserrent les liens de confiance établis. Ils deviennent également partie intégrante des documents légaux de la coopérative. Leur rédaction est une source d'apprentissage supplémentaire pour la directrice et le reste de l'équipe de direction. Chaque coopérative décide indépendemment de la manière dont son rapport annuel sera publié mais le budget doit respecter un format préalablement établi.

Durant les premiers mois, l'équipe de FUNDE rend visite à la coopérative et à ses membres chaque semaine et continue la formation de la directrice générale. A mesure que le temps passe, la fréquence de ces visites diminue. A l'issue de chaque visite, la directrice prépare un reçu, décrivant la nature des services rendus. Ce reçu détient une valeur symbolique autant que pratique. C'est non seulement un excellent outil permettant à FUNDE de suivre et de controler les déplacements et les performances de ses employées, mais encore, cela procure un pouvoir certain à la coopérative: Elle peut décrire et évaluer les services reçus, découvrant et soulignant ainsi ses droits à un service de qualite. Enfin, cette action contribue à renforcer son indépendance et son autonomie.

FUNDE limite la durée de son assistance à trois ans mais attend d'une coopérative qu'elle soit quasi-autonome au bout de deux ans. La Fondation ne versera pas plus de 10.000 Dollars US à la coopérative lors de sa première année d'activité, une restriction qui ne tient pas tant au fait que la coopérative ne possède pas les aptitudes nécessaires à gérer cet argent qu'aux difficultés rencontrées par FUNDE pour trouver plus de capitaux. Cependant, FUNDE ne cherche pas à devenir une source de subventions. Par contre, elle essaye d'être un intermédiaire actif entre les banques et les coopératives, se portant garante des emprunts négociés. Cela se produit déjà pour un certain nombre de coopératives parmi les mieux établies. Lorsqu'elle devient financièrement autonome, une coopérative peut acheter les services de FUNDE comme, par exemple, les sessions de formation, au lieu de les recevoir gratuitement.

Les principales étapes dans la croissance d'une coopérative interviennent en général comme suit: 
- Après deux ans: La coopérative a remboursé les premiers prêts accordés par FUNDE et peut assurer elle-même le salaire de sa directrice.

- Après quatre ans: Elle peut contracter des prêts auprès d'institutions financières extérieures avec la garantie de FUNDE.

- Après cinq ans: Elle peut payer pour l'assistance technique et tout autre service offert par FUNDE. Les prêts accordés par la Fondation sont utilisés pour diversifier.

- Après sept ans: La coopérative fonctionne de facon entièrement autonome.

\section{Résultats}

Durant ses sept ans d'expérience depuis le premier prêt accordé, FUNDE a participé à l'installation de 58 coopératives. 15 d'entre elles, dont nous avons parlé dans ce rapport, ont été établies pour et par des vendeuses de marché. 90\% de leurs membres sont des femmes. La plupart des 43 autres, situées près des marchés centraux ou en ville, regroupent des fermes et de petites entreprises de fabrication et de détail. 50 à $80 \%$ de leurs membres sont aussi des femmes. FUNDE aide également d'autres genres de coopératives, spécialisées en agriculture et autres projets de développement. Elles comptent en général un pourcentage d'hommes plus élevé.

De 1975 à 1979, le total des épargnes réalisées par les 58 coopératives passa de 74.556 à 1.640.500 Dollars US. Le montant des prêts accordés en 1979 s'éleva à 1.273.700 Dollars US. Au bilan général, le total des actifs augmenta de 126.056 Dollars US en 1975 à 623.000 Dollars US en 1979. L'accessibilité des crédits a permis aux femmes de maintenir et d'élargir leurs entreprises. Cela a également réduit le côut de certains aspects des transactions commerciales inhérentes à leurs activités. Par exemple, une femme, dont les ressources en argent liquide restent limitées, devait se rendre fréquemment chez ses fournisseurs pour y acheter ses biens et ce, en petite quantité. Avec plus d'argent disponible, elle réduit le nombre de ses tournées et achète en plus grande quantité à des prix inférieurs. Les économies ainsi réalisées finissent par se répercuter au niveau de leurs clientèles. Les coopératives ont aidé leurs membres à diminuer le montant de leurs dettes, augmenté leurs possibilités d'investissements à court ou longterme et encouragé l'épargne. De plus en plus, les emprunts se traduisent en investissements à long-terme comme l'éducation, l'achat d'une maison ou l'amélioration des conditions d'habitation. Cette diversification dans l'usage des prêts transforme progressivement une simple coopérative en une association d'épargne et de crédit aux buts multiples.

Les premières femmes à s'inscrire travaillaient déjà dans le secteur commercial et possédaient une certaine expérience dans la gestion de l'argent. Par contre, beaucoup parmi les plus récemment inscrites conduisent leurs affaires de chez elles plutôt que de faire partie d'une structure organisée. Le fait de pouvoir obtenir des crédits leur offrit la possibilité de se lancer dans des activités génératrices de revenus et de devenir un élément actif dans le développement économique de leur pays.

Les coopératives ont également changé la nature des relations entre les institutions financières en place et les communautés. La plupart des coopératives bien implantées ont maintenant reçu des prêts de banques locales sans autres intermédiaires. Ces transactions demeurent souvent les premières jamais effectuées par de telles institutions avec des entreprises gérées par des communautés aux revenus faibles. Même si les banques perçoivent un taux d'intérêt commercial normal sur ces prêts, ces derniers reflètent dans un certain sens leur volonté d'investir dans la partie pauvre de la communauté car ils facilitent indirectement une meilleur distribution des revenus. Les prêts accordés aux vendeuses, par exemple, aident à réduire les inégalités entre hommes et femmes.

La mise en place de coopératives a aussi provoqué un flôt d'informations relatives à l'usage et aux besoins d'argent ainsi qu'au changement progressif de ses usages. Auparavant, ces informations ne circulaient que partiellement parmi les "prestamistas" et, à un moindre degré, dans les banques. Maintenant, elles sont regroupées au coeur d'un système qui jouit de la confiance de la communauté, possède un statut légal vis à vis d'autres structures administratives et peut jouer un rôle de pression sur d'autres entreprises commerciales. Les adhérentes peuvent ainsi analyser leur propre situation économique et celle de leur communauté, les autorisant à organiser et à se lancer dans de plus vastes projets au niveau social et politique. Les coopératives jouissent d'une situation où elles peuvent identifier et mener à bien des projets communautaires qui ne seraient normalement entrepris ni par le gouvernement, ni par des compagnies privées. Quelques exemples:

- Une coopérative travaille à la mise en place d'une structure de marketing centralisée dans une communauté à l'heure 
actuelle sous la dépendance de Managua.

- A Chichigalpa, la coopérative opère deux crêches de jour pour des enfants en dessous de l'age scolaire. Cela rend service aux mamans et permet aux enfants de rester en bonne santé et de se familiariser avec la vie et la discipline scolaire.

- A Granada, la coopérative a ouvert un dispensaire où les docteurs de la région offrent leurs services plusieurs matinées par semaine. Produits de soins et médicaments sont gratuits.

En d'autres termes, une solide coopérative, s'appuyant sur une base économique ferme, peut en faire résulter un pouvoir politique et social plus important et une légitimité.

Certains avantages offerts par les coopératives restent quelquefois moins visibles ou quantifiables. Leur retentissement au niveau individuel peut cependant être retraduit dans les compte-rendus ci-dessous:

- Une femme, agée de trente ans, mariée et mère de quatre enfants, membre d'une coopérative depuis plusieurs années, fabrique des vêtements qu'elle vend localement. Elle utilisa son premier emprunt de 500 Cordobas pour se rendre à Panama où elle trouva des tissus et des étoffes en plus grande variété et meilleur marché qu'au Nicaragua. Le montant total de ses emprunts s'élève à l'heure actuelle à 6.000 Cordobas mais ses revenus annuels sont supérieurs à ses dettes. Pour acheter ses matériaux, elle voyage maintenant non seulement à Panama mais aussi au Mexique.

- Une veuve, agée de 70 ans et faisant partie d'une coopérative depuis plusieurs années, souffrit d'une hémorragie cérébrale. Elle emprunta auprès de la coopérative pour payer ses frais médicaux. Sans ce prêt, elle aurait sans doute fait faillite. Elle n'entend pas grand-chose à l'argent mais effectue ses paiements en temps voulu et a confiance en la coopérative.

- Une directrice de coopérative de vingt ans, qui était responsable de 300 personnes dans une usine de vêtements, préfère travailler à la coopérative car elle n'a pas à rester debout toute la journée, aime diriger et rendre service aux gens. Elle prend particulièrement plaisir à expliquer aux autres ce qu'est une coopérative et leur montrer comment cela fonctionne.
Elle souhaite pouvoir former d'autres femmes aux responsabilités de direction d'une coopérative.

L'un des objectifs des coopératives d'épargne et de crédit pour les vendeuses de marché était d'élever le statut des femmes dans la communauté ainsi que d'améliorer leur situation économique. Les bénéficiaires décrivent leurs nouveaux rôles et responsabilités de la manière suivante:

- Elles contrôlent mieux leurs destinées.

- Elles exercent plus d'influence dans les décisions du ménage.

- Elles ont acquis une connaissance solide de leurs droits aux services et au soutien de la communauté.

- De nombreuses femmes qui, auparavant, portaient leurs produits dans des paniers. louent maintenant des étales et disposent de leur espace propre.

- Elles ont les moyens d'investir dans l'éducation de leurs enfants.

- Avec une fierté toujours plus grande de leur profession, elles encouragent leurs filles à devenir vendeuses de marché.

- Elles entrevoient des moyens d'améliorer et de transformer leurs communautés en un environnement bon et propice à la croissance de leurs enfants.

L'une des raisons pour permettre aux femmes l'accès au crédit et à l'investissement est de leur donner une voix dans la vie de la communauté. Les coopératives des vendeuses de marché expriment leurs points de vue d'une manière tres importante. La plupart des projets qu'elles ont entrepris pour la communauté aident et renforcent leur rôle dans la vie domestique: Crêches, santé, eau potable dans les écoles, etc. Grâce à leur solide base économique, les vendeuses de marché peuvent s'assurer que leurs besoins prioritaires sont activement pris en considération. On peut se poser la question de savoir si une coopérative d'hommes aurait pu obtenir des résultats similaires.

\section{Leçons à Retenir}

On ne peut mettre en place des coopératives d'épargne et de crédit n'importe où et n'importe comment. Le modèle développé par FUNDE est la deuxième tentative de la Fondatión après un premier essai infructueux. Cette seconde tentative réussit car elle a tenu compte de plusieurs leçons apprises: 


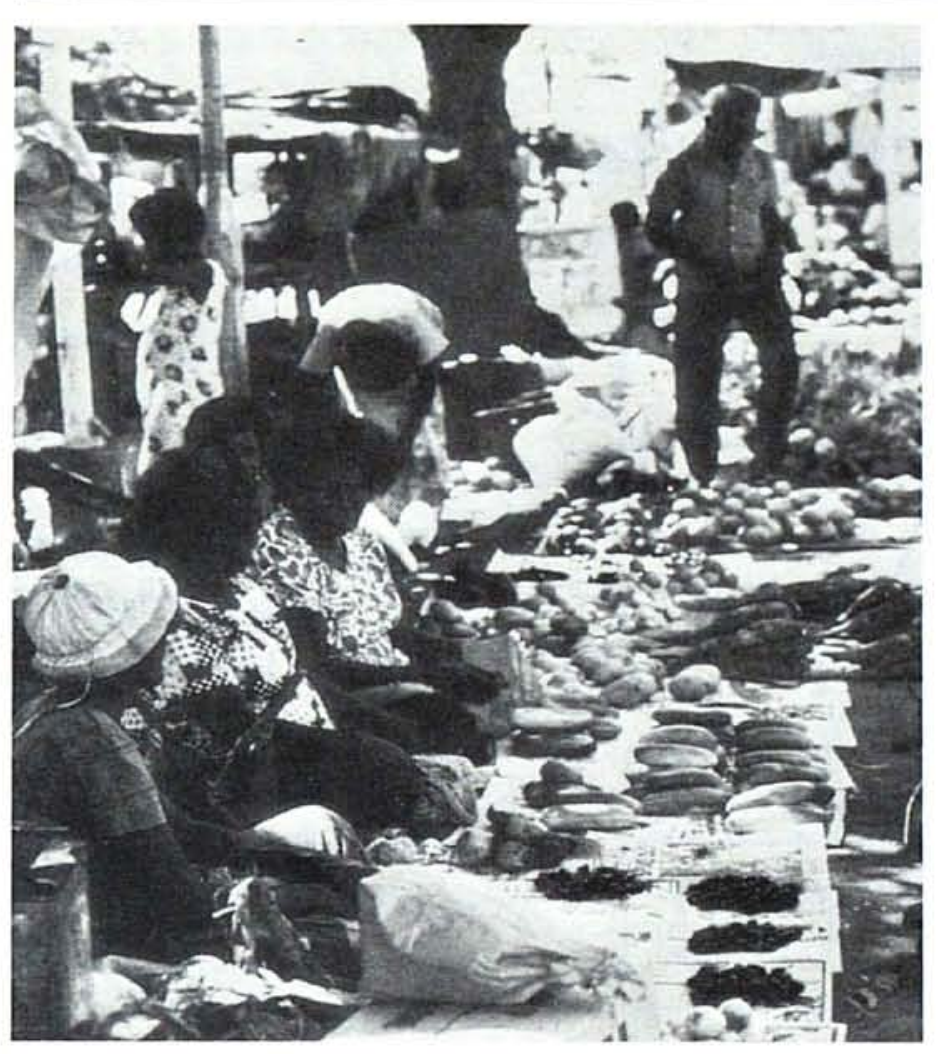

1. Le prix des parts que les adhérentes doivent acquérir au moment de leur inscription est suffisemment bas pour permettre à celles dont les revenus sont faibles de participer. Même les femmes pauvres peuvent économiser et mettre de côté, si ce n'est de l'argent, du moins quelque chose qu'elles peuvent par la suite revendre pour de l'argent (du riz, par exemple). II est important pour la coopérative de rester flexible en ce qui concerne les sommes reçues de femmes aux faibles revenus. Si les petits montants qu'elles peuvent économiser ne sont pas traités sérieusement, elles se désintéresseront du projet. FUNDE s'aperçut que les vendeuses de marché choisissaient d'emprunter auprès des "prestamistas" car elles pouvaient les rembourser en petite quantité chaque jour. De fréquents petits paiements semblent accélérer la vitesse avec laquelle les remboursements sont complétés car ils éliminent les tentations de dépenser cet argent ailleurs. Un système d'emprunt et de crédit, s'il veut fonctionner avec succès, se doit de pouvoir adapter ses plans de paiements et de versements en fonction des possibilités de ses participantes.

2. Un système de crédit en coopérative doit avoir un constituant, tel les vendeuses de marché, qui éprouve un besoin immédiat de ses services. Beaucoup de femmes, travaillant dans l'agriculture ou le petit com- merce, sont déjà à la recherche de crédits. Elles deviennent souvent les premières adhérentes et meneuses des coopératives et y attirent les autres femmes. Avant que celles plus pauvres et peu actives économiquement se lancent dans un tel projet, elles doivent pouvoir constater comment les besoins d'un groupe de femmes plus en vue sont adressés.

3. L'analyse des risques présentés par une adhérente qui souhaite obtenir un prêt doit être entreprise par ses associées, des femmes de la même communauté et engagées dans le même type d'activités, car elles peuvent évaluer plus facilement l'intégrité de la candidate. De cette manière, le nombre des remboursements, sans nécessité de garanties, peut être élevé. Les procédures traditionnelles d'emprunt, proposées par les institutions financières de la communauté, y compris les prêteurs, exigent souvent des garanties. C'est un handicap pour celles qui pourraient effectuer leurs remboursements mais n'ont aucun actif tangible à soumettre en garantie. Le système adopté et mis en place par FUNDE dans les coopératives renforce un sens des responsabilités au niveau des communautés en regard d'un taux de remboursement rapide. Mais surtout, il permet aux défavorisées de faire de l'argent sans en avoir au préalable

4. Un organisme qui décide d'implanter des coopératives d'emprunt et de crédit n'a pas besoin de capitaux importants (peutêtre 10.000 dollars US, dont 5.000 en subventions et $\mathbf{5 . 0 0 0}$ en prêts). II doit cependant vouloir et être capable de s'engager à long-terme dans le développement d'initiatives et d'idées chez des femmes dont les revenus sont limites. Cet organisme doit pouvoir consacrer suffisemment de temps, de un à quatre ans, s'il veut développer ce genre de projet lentement. II doit être compétent dans l'organisation et la conduite de stages de formation préparant les femmes à la gestion de leur argent. II doit pouvoir procurer une solide assistance technique et assurer un soutien moral et pratique constant durant les premières années. Parmi les organismes qui entreprennent de se lancer dans des projets d'action sociale, certains pourraient confier à la charge de leurs employés la promotion du concept des coopératives et utiliser leurs subventions pour implanter des systèmes d'emprunt et de crédit et les faire fructifier Si l'on se réfère à l'expérience de FUNDE, la 
plupart des coopératives peuvent rembourser leurs dettes en deux ou trois ans et rares sont les projets de développement où un organisme peut s'attendre à recouvrer son investissement initial.

5. La mise en place d'une coopérative représente essentiellement la construction d'un contrat social pour et par ses membres et augmente le niveau de confiance des individus. La présentation et l'explication du concept prennent du temps. Cela ne peut s'accomplir qu'avec un matériel solide, de nombreux encouragements et discussions et des supports visuels efficaces qui feront comprendre facilement aux femmes, illettrées ou non, l'importance de leur rôle dans la vie et la réussite de la coopérative.

6. La coopérative doit pouvoir obtenir des résultats rapides et tangibles. $\mathrm{Par}$ exemple, FUNDE réussit généralement à compléter les formalités administratives et légales indispensables et à ouvrir les bureaux de la nouvelle coopérative en l'espace de trois mois. Les adhérentes sont autorisées à déposer de l'argent sur leurs livrets dès que cela est légalement possible. Les premiers prêts sont accordés dans les six mois. Au début, l'ensemble des réunions sont ouvertes à toutes. Les cartes d'adhérents, les posters muraux et les rapports annuels sont des preuves tangibles de participation, de croissance et de légalité qui renforce la crédibilité de la coopérative.
Il existe de nombreuses sources d'information pour les personnes intéressées dans le développement de coopératives. D'autres projets similaires à ceux exposés dans ce rapport sont mentionnés dans l'appendice III.

FUNDE a etabli un centre de recherche et de développement qui sera heureux de vous communiquer de plus amples informations sur ses programmes de mise en place de coopératives. Ecrivez à:

Lic. William Baez

FUNDE

Apartado 2598

Managua, Nicaragua. 


\section{Appendice I}

\section{ORGANISATION D'UNE COOPÉRATIVE}

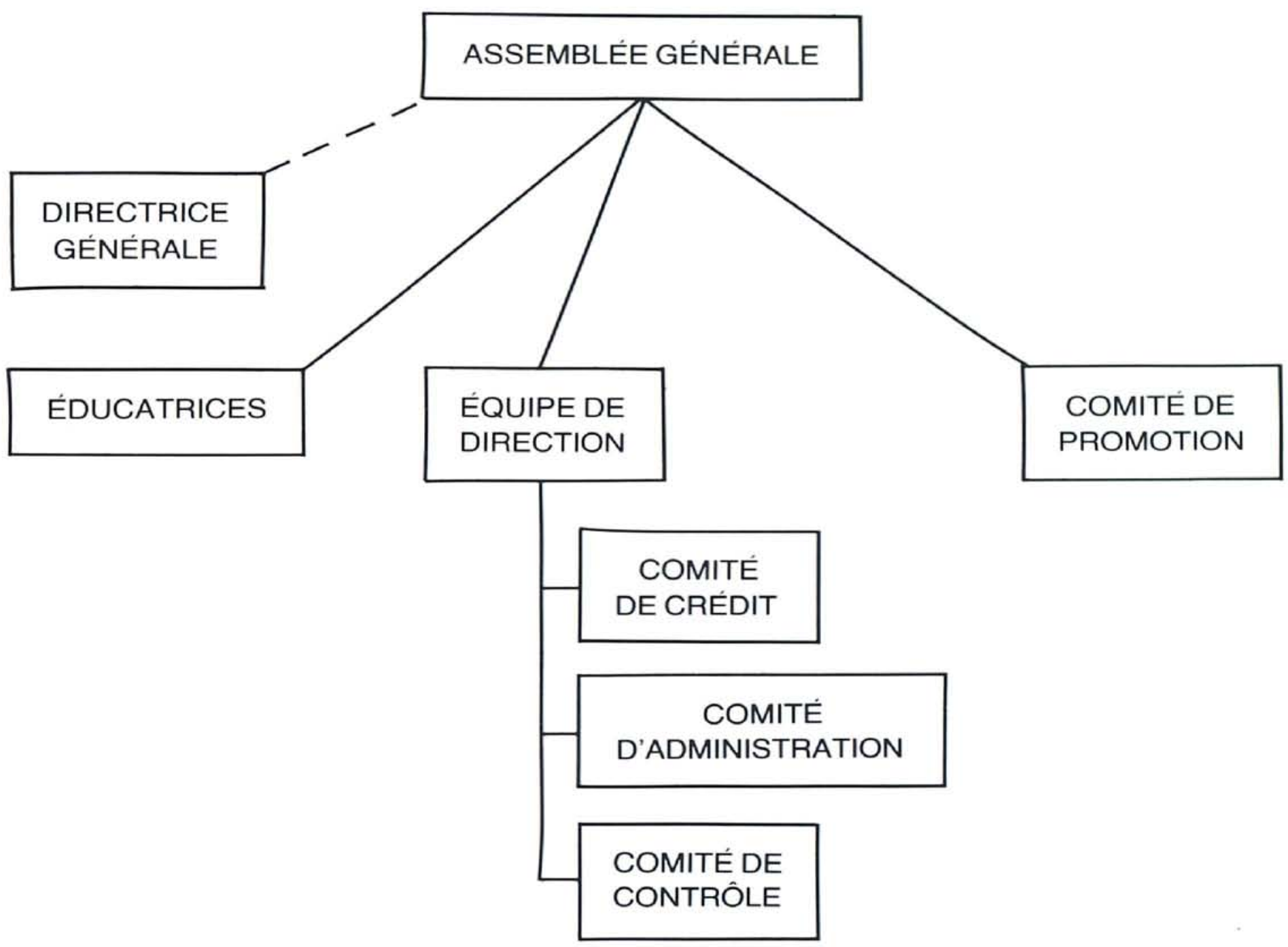

Assemblée Génerale: Tous les membres font partie de l'Assemblée Générale et sont invités à une réunion annuelle où l'on présente un rapport des activités de la coopérative et approuve les transactions financières et fiscales. Les onze directrices y sont également élues

Directrices: Onze membres élues lors de l'assemblée générale annuelle et formant le Comité de Crédit. le Comité d'Administration et le Comité de Contrôle.

Comité de Crédit: Se réunit chaque semaine pour analyser les demandes de prêts. Accorde ou rejette les demandes et réajuste l'échelonnement des paiements si nécessaire.

Comite d'Administration: Se réunit chaque semaine et veille au bon fonctionnement des opérations S'assure de la bonne marche des bureaux et de l'enregistrement systématique des activités quotidiennes. Surveille également le travail effectué par la directrice générale.

Comité de Contrôle: Se réunit chaque semaine pour vérifier toutes les transactions fiscales en cours. les prêts accordés et les décisions administratives.

Educatrices: Les éducatrices sont sélectionnées parmi les adhérentes et ont la charge de la formation des nouvelles. De plus, elles doivent assurer la formation continue de toutes, en liaison avec FUNDE

Directrice Generale: Engagée par la coopérative. elle est responsable des lieux. Elle ouvre et ferme les bureaux et gère quotidiennement les demandes de prêts et les remboursements.

Comite de Promotion: Les membres du Comité de Promotion sont élues par les adhérentes. Elles parlent de la coopérative avec le reste de la communauté. encouragent les nouvelles inscriptions et recherchent les nouveaux rôles que la coopérative pourrait remplir au sein de la communauté 


\section{Appendice II}

FUNDE rédigea une simple brochure expliquant le concept d'une coopérative à d'éventuels membres. La première page de cette brochure est reproduite ci-dessous. La traduction en est: "Qu'est-ce qu'une coopérative? Une coopérative est l'association d'un groupe de personnes qui partagent les mêmes problemes et qui décident volontairement de s'organiser pour s'entraider et rendre service à leur communauté. Comment se forme une coopérative? Une coopérative est formée par la volonté propre d'un groupe de personnes".

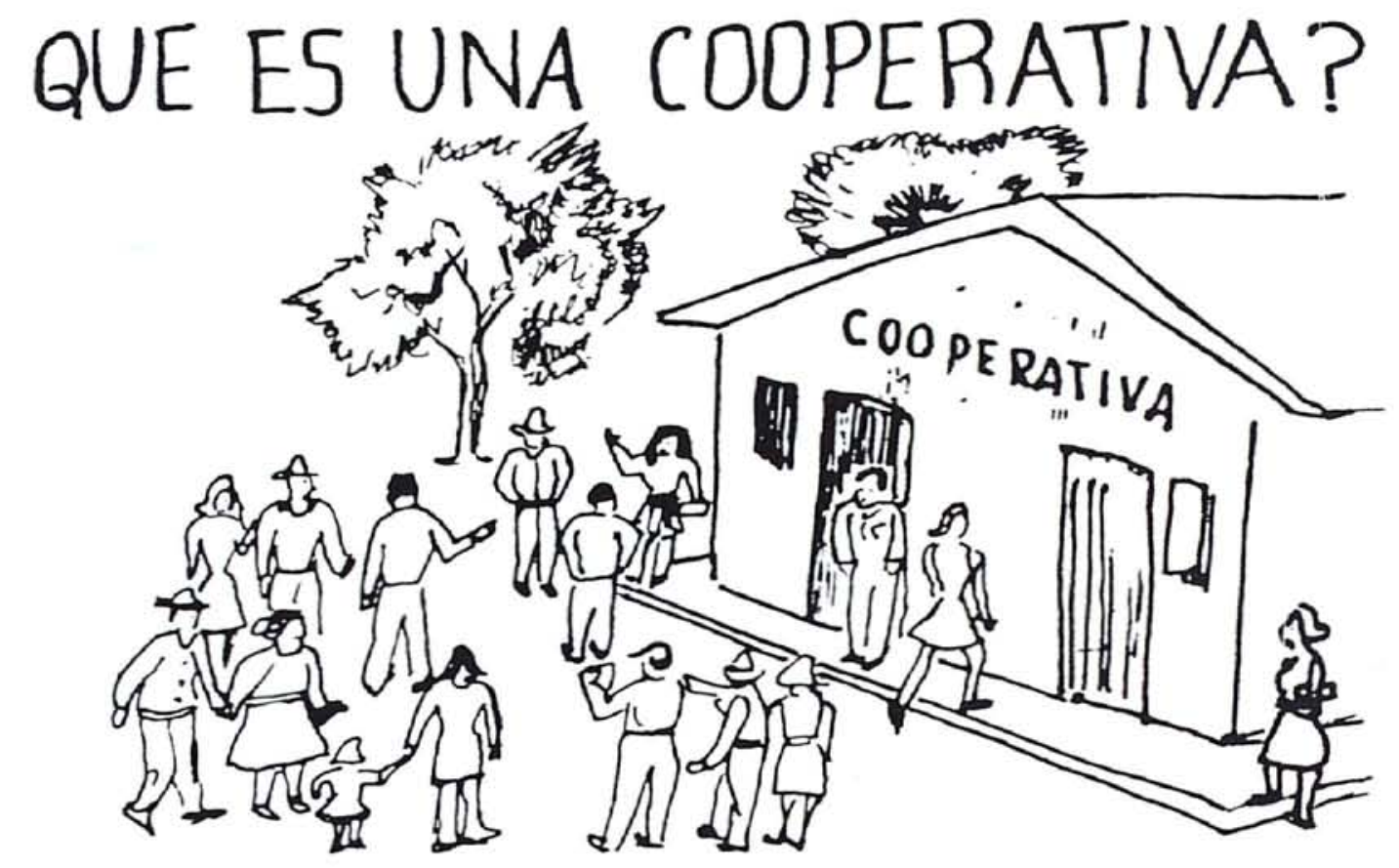

UNA COOPERATIVA ES LA UNION DE UN GRUPO DE PERSONAS QUE TIENEN LOS MISMOS PROBLEMAS Y QUE SE ORGANIZAN VOLUNTARIAMENTE PARA SERVIRSE A SI MISMO O A LA COMUNI DAD.

\section{COMO SE FORMA UNA CODPERATIVA?}

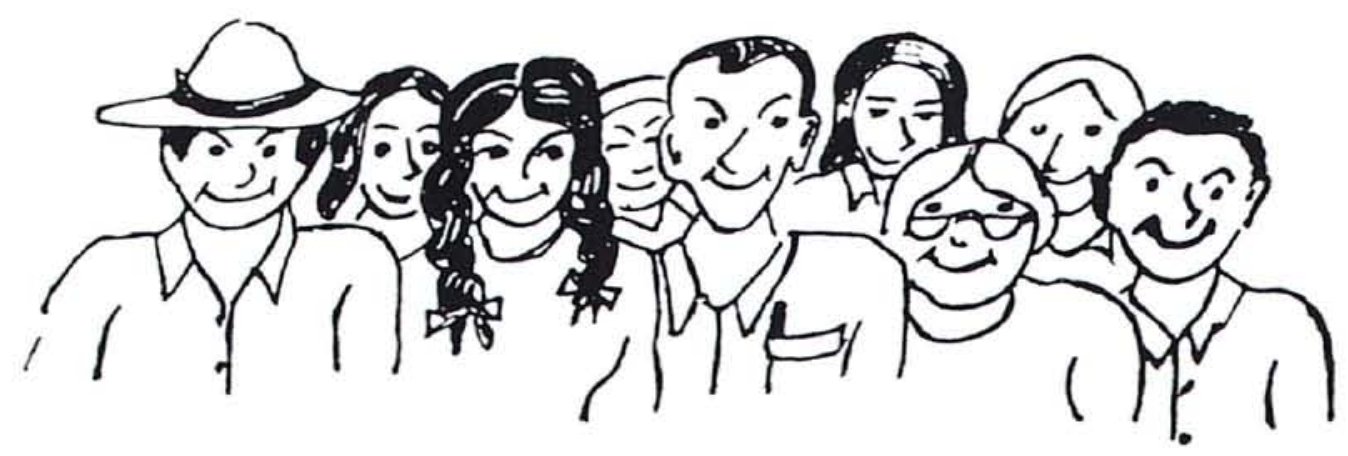

SE FORMA POR LA VOLUNTAD PROPIA DE UN GRUPO DE PERSONAS. 


\section{Appendice III}

Le développement de coopératives de crédit pour des femmes, où elles peuvent emprunter et épargner et qui joue un rôle d'action sociale, peut se produire dans divers environnements. En voici deux exemples:

\section{Self-Employed Women's Association of Ahmedabad (I'association des femmes travaillant à leurs comptes, Ahmedabad, Inde, SEWA).}

SEWA est une association commerciale de 12.000 Indiennes aux revenus faibles et travaillant à leurs comptes. Elles sont haleuses de chariots, vendeuses de légumes, chiffonières, revendeuses de vieux vêtements, fabricantes de couvertures et petites commerçantes. 93\% sont illettrées et $97 \%$ habitent dans des bidonvilles. La plupart ne sont pas propriétaires de leurs équipements (étales de vente, chariots, etc.).

Quand SEWA fut créée, la majorité de ces femmes étaient fortement endettées auprès de leurs prêteurs et de leurs fournisseurs en gros. Pour adhérer à SEWA, une femme doit payer 3 Roupies (environ une journée de salaire). Quand c'est chose faite, elle peut acheter une action à la Banque des Femmes pour 10 Roupies (1,30 Dollars US). La banque accorde à ses actionnaires des prêts s'élevant de 250 à 1000 Roupies (33 à 132 Dollars US). Cet emprunt doit être remboursé en vingt mois avec des paiements égaux chaque mois. La banque perçoit $10 \%$ de commission mais elle octroie un abattement si le prêt est remboursé en temps voulu et une pénalité, dans le cas contraire. Une étude effectuée sur 2000 participantes révèle que $44 \%$ honorent leurs paiements en temps voulu. $43 \%$ manquent de 3 à 6 paiements et $13 \%$ seulement en manquent plus de 6 .

II existe un rapport plus détaillé de ce projet dans "Les Femmes Dans Une Economie En Voie de Développement-De La Dissociation A La Réhabilitation: Rapport Sur Une Expérimentation Pour Promouvoir Le Travail Individuel Dans Un Environnement Urbain", par Devaki Jain, publié par la Commission Indienne de Recherches en Sciences Sociales, IIPA Hostel Building, Indraprastha Estate, Ring Road, New Delhi I, Inde, 1976. On peut se procurer de plus amples informations auprès de: Mrs. Ela R. Bhatt, Chief, Women's Section, Textile Labor Association, Ahmedabad, Inde.

\section{L'Association de Crédit pour Petites Entreprises est le second projet de dé- veloppement urbain à San Salvador, El salvador.}

C'est un projet gouvernemental, financé par la Banque Mondiale, dont le but est d'améliorer l'environnement urbain grâce à un système qui met en valeur et élargit les services de base et le soutien offerts aux petites entreprises locales. Les foyers supportés économiquement par les femmes représentent plus de $40 \%$ des participants au projet. De plus, les recherches effectuées dans la région montrent que les femmes sont entièrement responsables des finances familiales, quelque soit leur situation maritale et c'est pour cette raison que le projet fut lancé. II aide les femmes à augmenter leur productivité et à percevoir des revenus en argent liquide. II leur permet également de devenir moins dépendantes de l'aide de leurs filles pour leurs entreprises et ces dernières peuvent ainsi aller à l'école.

Les femmes ne soumettent pas directement à l'organisme municipal les demandes de prêts individuelles mais présentent leurs requêtes par l'intermédiaires de groupes de solidarité organisés. Elles doivent en premier lieu appartenir à un tel groupe dont les membres examinent leurs demandes. La responsabilité du remboursement revient ensuite au groupe entier. Des qu'il arrive à une décision favorable, il transmet le dossier à l'organisme financier municipal qui octroie les montants demandes et reçoit les paiements.

Durant la première année du projet, 85\% des demandes furent soumises par des femmes possédant leur propre entreprise. La majorité des prêts s'élevèrent de 200 à 300 Dollars US et $22 \%$, de 400 à 500 Dollars US. $60 \%$ des emprunts sont contractés pour maintenir des boutiques telles que des magasins d'alimentation familiale. $25 \%$ affectent la fabrication de vêtements pour femmes et enfants. $10 \%$ viennent en aide aux artisans et fabricants des produits artisanaux. Enfin, 5\% sont accordés à des entreprises de manufactures, tailleries, cordonneries et réparations. La 
première année, $97 \%$ des paiements furent honorés. Une étude des entreprises des demandeuses de prêts révéla que la plupart avaient augmenté en moyenne de $40 \%$ le volume de leurs activités.

Pour de plus amples informations, se référer à: "Crédits pour les Femmes de Milieu Rural: Faits et Leçons", rapport rédige par Myra Buvinic, Jennefer Sebstad et Sondra Zeidenstein. On peut se le procurer auprès de: Women in Developpement Office, Agency for International Development, State Department, Washington. D.C. 20523. 
Maquette: John Cotterman

Typographie: Village Type \& Graphics

Photo de Couverture: Organisation des Nations Unies.

Imprimerie: Graphic Impressions, Inc.

Traduit de I'Américain par: Philippe Bette

Nous attendons vos remarques, commentaires et idées de projets à publier dans les prochains numéros de SEEDS. Si vous souhaitez recevoir des exemplaires supplémentaires de ce numéro ou faire partie de nos abonnés, n'hésitez pas à nous écrire. Envoyez votre courrier à:

Ann Leonard, éditeur SEEDS

P.O. Box 3923

Grand Central Station

New York, New York 10163 U.S.A. 


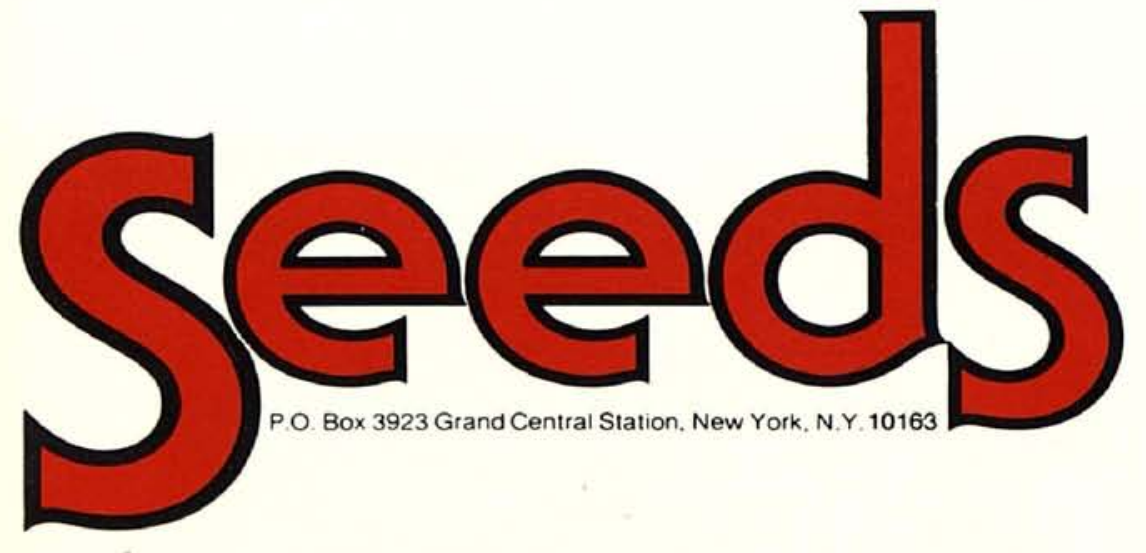

